\title{
Modelling Tourism in Australia Based on Periodic Autoregressive Time Series Models
}

\author{
Ezra Precious Ndidiamaka ${ }^{1}$, Okonta Charles Arinze ${ }^{2}$, Okoro Udu Ukpai ${ }^{2}$ \\ ${ }^{1}$ Department of Statistics, Faculty of Physical Sciences, University of Nigeria, Nsukka, Nigeria \\ ${ }^{2}$ Department of Maths/Statistics, School of Science, Akanu Ibiam Federal Polytechnic, Unwana, Nigeria \\ Email address: \\ precious.ezra@unn.edu.ng (E.P. Ndidiamaka),okorouduukpai@gmail.com (O. U. Ukpai), \\ okontacharlse1973@gmail.com (O. C. Arinze)
}

To cite this article:

Ezra Precious Ndidiamaka, Okonta Charles Arinze, Okoro Udu Ukpai. Modelling Tourism in Australia Based on Periodic Autoregressive Time Series Models. Pure and Applied Mathematics Journal. Vol. 10, No. 4, 2021, pp. 96-103. doi: 10.11648/j.pamj.20211004.12

Received: July 27, 2021; Accepted: August 9, 2021; Published: August 19, 2021

\begin{abstract}
Tourism has become very important in international commerce and also represents one of the main sources of income for some developing countries in the world. It is the act of people travelling and staying in a place outside their usual environment for leisure, business or other purposes, and this may include sightseeing, camping, retreats, etc Tourism is one of the key economic growth contributors and has contributed towards complete growth and development of Australia by bringing numerous economic value and benefits to her, and also building her brand value, image and identity. This paper seeks to generate a periodic autoregressive PAR model that could be used to make reliable forecast for tourism in Australia. Periodic autoregressive models are for seasonally observed data, particularly quarterly and monthly. Its' parameters take different values across the seasons. The data used in this work was extracted from the official website of the Australian Bureau of Statistics (ABS), (www.abs.gov.au). It consists of monthly historical data of the number of short term visitors in Australia from January 1998 to December 2017 and was analysed using R- statistical software. The result revealed the order of the PAR model, and also verified that there is periodic variation (periodicity) in the tourism data. It was also verified that there is existence of single unit root and periodic integration which led to the fitting of periodic integrated autoregressive (PIAR(2)) model as the suitable model for the Australian tourism data. Finally, the residual generated from the model was subjected to statistical test and it showed a white noise behavior. Based on these findings, it is concluded that periodic autoregressive time series model can be used to generate reliable forecast for Australian tourism data. Further research on the stochastic nature and seasonality of Australian tourism data was recommended.
\end{abstract}

Keywords: Periodicity, Periodic Autoregression, Residual, Tourism, Likelihood Ratio

\section{Introduction}

Tourism is the act of people travelling and staying in a place outside their usual environment for leisure, business or other purposes, and this may include sightseeing, camping and retreats [21]. Tourism may also involve people travelling to a place to learn about the history or culture of the destination or even to learn about the people who live there; people from a particular region travelling to a different region to have a taste of an entirely different environment (for example, people from a colder region travel to a warmer region to relax in the sun); people travelling in order to get engaged in an event or activity which they could not readily access at home; or people visiting families and friends in another region or going on some form of pilgrimage etc. [21]

Tourism helps improve the economy of a place. This can be seen majorly in areas such as transportation (roads, airlines, railways and water transportation), and accommodation (hotels, camping grounds, parks, restaurants, café and bars). Tourism also attracts investors to a particular place thereby boosting the foreign investment of that region.

Over the years, tourism has seen a continuous growth and increasing diversification to emerge as the world's fastest growing economic sector for developed countries [1, 2]. Currently, the business volume of tourism equals or even surpasses those of oil exports, food production or automobile merchandise. Tourism has become very important in international commerce and also represents one of the main 
sources of income for many developed and developing countries in the world including Australia [1, 2].

In Australia, tourism is an important component of their economy. Australian tourism satellite account recorded that there were about 7.4 million tourists in Australia, specifically in the 2015 calendar year [1]. In the same period, over 580,800 were employed in Australia by tourism alone, and this constitutes about $5 \%$ of the Australian workforce as at then. Also, according to the Australian tourism satellite account, tourism represented $3.0 \%$ of the Australia's GDP which was evaluated at about $\$ 47.5$ billion [2]. Also, as at June 2019 , it was reported that tourism increased by $3 \%$ having a record of 8.6 million tourists.

The objective of this paper is to model tourism in Australia using Periodic Autoregressive model (PAR). This involves fitting a periodic autoregressive model of order $p, \operatorname{PAR}(p)$, selecting the periodic autoregressive lag order parameter, testing for periodicity (periodic variation) using the data, testing for unit root and periodic integration, fitting a periodically integrated autoregressive model PIAR, and making forecast.

According Osborn and Osborn and Smith, the introduction of periodic models into economics dated back to the late $1980[16,15]$. Then, the focus was on describing trending consumptions and income data and the use of periodic models for out-of-sample forecasting. These studies did not include a formal analysis of the type of trends, simply because the relevant tools were developed in the mid-1990s $[3,4]$. Other new developments were extensions to multivariate periodic models for trending data and formal model selection strategies.

Over the years, the study on periodic autoregressions has improved significantly. It has been used frequently in hydrological and environmental studies but has been recently introduced into the economic and other range of studies [17, 18]. In [18], the study focused on out of sample forecasting of quarterly UK consumption series. Since that study, the works on periodic models have developed substantially. There have appeared several studies on evaluating forecasts from PAR models $[16,22,11,12,8]$ and they vary in conclusions.

It is well known that some of the economic time series display stochastic trends. Moreso, when dealing with stochastic seasonal time series data, seasonality may exist as well. Hylleberg stated that seasonality is the systematic although not necessarily regular, intra-year movement caused by changes of weather, calendar and timing of decisions, directly or indirectly through the production and consumption decisions made by agents of the economy [14]. Franses reviewed recent developments in econometric modeling of economic time series with seasonality [8, 9]. The prime focus was on economic models which incorporate explicit description of seasonal variation instead of moving from one variation using a seasonal adjustment method. The review centered on developments in seasonal unit root model and in-periodic parameter models, both in univariate and multivariate contexts. Franses and Paap stated that there are various approaches to modeling and forecasting seasonal time series [8-10]. According to them, one approach builds on the work of Box and Jenkins (1970) which relies on moving average models for double differenced time series called Seasonal Auto-regressive Integrated Moving Average (SARIMA) models. Second approach assumes that seasonal time series can be decomposed into trend, cycle, seasonal and irregular components [11]. Third approach questions the adequacy of the double differencing filter in SARIMA models and mainly addresses the issue of how many unit roots should be imposed in autoregressive models [14]. Finally, fourth approach assumes that seasonal variation is best described by allowing the parameters in an autoregression to vary with the seasons, that is, the so-called Periodic Autoregression (PAR). Lopez-de-Lacalle stated that when the component of time series trend and seasonality, do not evolve independently, traditional differencing filter may not be suitable, but can be handled using a seasonally varying parameters and a periodic differencing filter which is the periodic autoregressive time series models [15]. Ursu and Duchesne suggested that the method of moments based on Yule-Walker equations and the least squares method in the univariate case of (Franses and Paap, 2004) are efficient to estimate PAR models [21]. According to Hipel and McLeod, when the seasonal data and the model for each season are used rather than the annual data and the associated model, significant gain in parameter efficiency can be achieved [13]. It was indicated however that the main problem in PAR modeling relies on the number of parameters that need to be estimated which increases with the choice of the season for the data. Moving from quarterly surveys to monthly, data increases both the number of models and the number of parameters to be estimated. To obtain parsimonious models, it is of interest to study situations in which linear constraints on the parameters of a given season are introduced. Eugen and Jean-Christopher developed a robust modeling approach to identify and to estimate periodic autoregressive (PAR) model in the presence of additive outliers [5-7]. Since the least squares estimators are not robust in the presence of outliers, they suggested a robust estimator based on residual autocovariances. A genetic algorithm with Bayes information criterion (BIC) was used to identify the optimal PAR model. The method was applied to average monthly and quartermonthly flow data (1959-2010) for the Garonne River in the southwest of France. Results showed that the accuracy of forecasts was improved in the robust model with respect to the unrobust model for the quarter-monthly flows [22].

\section{Materials and Methods}

This chapter discusses the methods of data analyses.

\subsection{Materials}

This paper makes use of the secondary data extracted from the official website of the Australian Bureau of Statistics (ABS), (www.abs.gov.au). It consists of monthly historical data of the number of short term visitors in Australia from January 1998 to December 2017. 


\subsection{Methods}

The following models were used to analyze the data in this work.

\subsubsection{Periodic Autoregressive PAR Model}

Periodic autoregressive (PAR) models are meant for seasonally observed time series data, usually quarterly and monthly data. Considering a univariate time series $\left\{X_{r d+s}\right\}$ which is periodic and is observed monthly for $r$ years, then such periodic time series $\left\{X_{r d+s}\right\}$ having zero mean and finite variance is said to have periodic autoregressive model of order $p$ with $d>0$ and denoted by $P A R_{d}(p)$ if it satisfies the following formula,

$$
\begin{gathered}
X_{r d+s}=\varphi_{s, 1} X_{r d+s-1}+\varphi_{s, 2} X_{r d+s-2}+\cdots+\varphi_{s, p} X_{r d+s-p}+\varepsilon_{r d+s} \\
X_{r d+s}=\sum_{i=1}^{p} \varphi_{s, i} X_{r d+s-i}+\varepsilon_{r d+s}
\end{gathered}
$$

Where, $\varepsilon_{r d+s} \sim \operatorname{PWN}\left(0, \sigma_{s}^{2}\right), d$ is the number of seasons, $\varphi_{s, i}, i=1,2, \cdots, p$ are the model's parameters for seasons $s=1,2, \cdots, d, r=0,1,2, \cdots, N-1$

The formula in (1) can also be written in autoregression operator of order $\mathrm{p}$ form as

$$
\Phi_{s, i}(B) X_{r d+s}=\varepsilon_{r d+s},
$$

Where, $\Phi_{s, i}(B)=1-\varphi_{s, 1} B-\varphi_{s, 2} B^{2}-\cdots-\varphi_{s, p} B^{p}$.

Note that the $P A R_{d}(p)$ model considers different $A R(p)$ models for different seasons, and the process described in (1) is non-stationary as the autocovariance and autocorrelation are time-varying within the year. However, the process can be converted to time-invariant form by rewriting the process in (1) as a d-dimensional vector autoregression $\operatorname{VAR}(\mathrm{P})$ model written as

$$
\Phi_{0} Y_{r}=\Phi_{1} Y_{r-1}+\Phi_{2} Y_{r-2}+\cdots+\Phi_{P} Y_{r-P}+\epsilon_{r}
$$

Where, $Y_{r}=\left(X_{r d+1}, \cdots, X_{r d+d}\right)^{\prime}$

$\Phi_{0}$ is $(d \times d)$ lower triangular matrix with unit elements on the diagonal such that

$$
\Phi_{0}=\left(\begin{array}{ccccc}
1 & 0 & \cdots & 0 & 0 \\
-\varphi_{2,1} & 1 & \cdots & 0 & 0 \\
\vdots & \vdots & \cdots & \vdots & \vdots \\
-\varphi_{d, d-1} & -\varphi_{d, d-2} & \cdots & -\varphi_{d, 1} & 1
\end{array}\right)
$$

$\Phi_{i}{ }^{\prime} s, i=1,2, \cdots, P$ are $(d \times d)$ matrices

$$
\Phi_{i}=\left(\begin{array}{ccccc}
\varphi_{1, i d} & \varphi_{1, i d-1} & \cdots & \varphi_{1,(i-1) d+2} & \varphi_{1,(i-1) d+1} \\
\varphi_{2, i d+1} & \varphi_{2, i d} & \cdots & \varphi_{2,(i-1) d+3} & \varphi_{2,(i-1) d+2} \\
\vdots & \vdots & \cdots & \vdots & \vdots \\
\varphi_{d,(i+1) d-1} & -\varphi_{d,(i+1) d-2} & \cdots & -\varphi_{d, i d+1} & \varphi_{d, i d}
\end{array}\right)
$$

and, $\in_{r}$ is $(d \times 1)$ vector white noise, $\in_{r}=\left(\begin{array}{c}\varepsilon_{r d+1} \\ \vdots \\ \varepsilon_{r d+d}\end{array}\right)$

(4) gives rise to

$$
Y_{r}=\Phi_{0}^{-1} \Phi_{1} Y_{r-1}+\Phi_{0}^{-1} \Phi_{2} Y_{r-2}+\cdots+\Phi_{0}^{-1} \Phi_{P} Y_{r-P}+\Phi_{0}^{-1} \in_{r}
$$

\subsubsection{PAR model Parameter Estimation}

To estimate the parameters of a $\operatorname{PAR}_{d}(\mathrm{p})$ model, Franses and Paap recommend the use of seasonal dummy variables $D_{s, r d+s}$ which are equal to 1 for corresponding season $s$ and zero otherwise [10]. The parameters of the $P A R_{d}(p)$ model in (1) can be estimated by applying the regression model as below

$$
X_{r d+s}=\sum_{s=1}^{d} \varphi_{s, 1} D_{s, r d+s} X_{r d+s-1}+\sum_{s=1}^{d} \varphi_{s, 2} D_{s, r d+s} X_{r d+s-2}+\cdots+\sum_{s=1}^{d} \varphi_{s, p} D_{s, r d+s} X_{r d+s-p}+\varepsilon_{r d+s}
$$

Note that under normality of the error term $\varepsilon_{r d+s}$, the estimators of the parameters $\varphi_{s, i}, i=1,2, \cdots, p$ and $s=1,2, \cdots, d$ are obtained from ordinary least square (OLS) method of estimation of (6) since $P A R_{d}(p)$ is an extension of $A R(p)$.

\subsubsection{PAR Model Order of Selection}

Franses and Paap recommends the use of Bayesian

$$
\hat{\varepsilon}_{r d+s}=\alpha_{s, 1} X_{r d+s-1}+\alpha_{s, 2 s} X_{r d+s-2}+\cdots+\alpha_{s, p} X_{r d+s-p}+\tau_{s} \hat{\varepsilon}_{r d+s-1}
$$

Where $\hat{\varepsilon}_{r d+s}$ are the estimated residuals of (6).

The formula for the Bayesian Information Criterion is given as
Information Criterion (BIC) in combination with diagnostic tests on residual autocorrelation to select the order $\mathrm{p}$ of a periodic autoregressive model [8]. This is because when dealing with periodic time series model, it is reasonable to test for periodic serial correlation in the residuals. This can be achieved using a standard F-test for the significance of the $\tau_{s}$ parameter in the auxiliary regression model as below
Bayesian Information Criterion:

$$
B I C=-2 \ln (\hat{L})+k \ln (n)
$$


Where, $k=$ number of estimated parameters in the model, $n=$ number of observations, $\hat{L}=$ estimated maximized value of the likelihood function of the model.

\subsection{Test for Periodic Variation in the Autoregressive Parameters}

Once a PAR model has been defined and its parameters estimated, it is necessary that the periodic variation (periodicity) in the autoregressive parameters to be checked. Following the model in (1), we perform an F-test for the null hypothesis of non-periodicity,

Ho: $\varphi_{s, i}=\varphi_{i} \quad \forall \quad s=1,2, \cdots, d \quad$ and $i=1,2, \cdots, p$. Boswijk and Frances (1996), gave the likelihood ratio LR test for the null hypothesis as

$$
L R=n\left[\ln \left(R S S_{0}\right)-\ln \left(R S S_{1}\right)\right]
$$

Where, $n=$ number of observations, $R S S_{0}$ and $R S S_{1}=$ residual sums of squares for $A R(p)$ and $P A R_{d}(p)$ respectively.

When the null hypothesis is accepted an $A R(p)$ is estimated, while if it is rejected, a $P A R_{d}(p)$ model is fitted. It is worthy to note that when $k$ seasonal intercepts are included in the model, the statistic follows an F-distribution with $(3 p, n-k(1+p))$ degrees of freedom.

\subsection{Test for Unit Root and Periodic Integration}

The characteristic matrix polynomial $\Phi(z)$ of VAR(P) of (4) is defined as $\Phi(z)=\Phi_{0}-\Phi_{1} z-\Phi_{2} z^{2}-\cdots-\Phi_{p} z^{p}$. The VAR(P) process $Y_{r}$ is stationary provided that the root of the characteristics equation are outside the unit circle in modulus, that is,

$$
|\Phi(z)|=0
$$

On the other hand, the process $Y_{r}$ can be said to be integrated if (10) has a unit root, that is, if it is the case $\alpha_{1} \cdot \alpha_{2} \cdot \alpha_{3} \cdots \alpha_{d}=1$, that is, $\prod_{i-1}^{d} \alpha_{i}=1$ assuming $\alpha$ 's is used to represent $\varphi$ 's in the characteristic equation (10), using $P A R_{d}(1)$ for instance.

The restriction is satisfied in two particular cases. The first case is when $\alpha_{, 1}, \alpha_{2}, \alpha_{3}, \cdots$, and $\alpha_{d}$ are either 1 or -1 , then the model is known as a PAR process for a I(1) time series, PARI with long run seasonal unit root 1 , or the seasonal unit root -1 . The second case is when the restriction $\alpha_{1} \cdot \alpha_{2} \cdot \alpha_{3} \cdots \alpha_{d}=1$ is fulfilled, the model is known as a periodically integrated AR process, PIAR.

To test the above restriction, to see whether the process is a PARI process with the long run seasonal unit root 1 , or the seasonal unit root -1 , or a PIAR process, we first estimate the unrestricted equation.

$$
X_{r d+s}=\sum_{s=1}^{d} \varphi_{s, 1} D_{s, r d+s} X_{r d+s-1}+\varepsilon_{r d+s}
$$

Then, by imposing the above restriction, we have the following restricted equation

$$
X_{r d+s}=\sum_{s=1}^{d-1} \varphi_{s, 1} D_{s, r d+s} X_{r d+s-1}+\left(\alpha_{1} \cdot \alpha_{2} \cdots \alpha_{d-1}\right)^{-1} D_{d, r d+s} X_{r d+s-1}+\varepsilon_{r d+s}
$$

which can be estimated by non-linear least squares.

The null hypothesis to be tested is

Ho: $\varphi_{s, i}=1$ for $s=1,2, \cdots,(d-1)$ or $H o: \varphi_{s, i}=-1$ for

$$
s=1,2, \cdots,(d-1)
$$

and the likelihood ratio test statistic in (9) is used to test the null hypothesis where $R S S_{0}$ and $R S S_{1}=$ residual sums of squares for unrestricted model and restricted model respectively.

When the null hypothesis is accepted PARI process is fitted, while if it is rejected, a PIAR process is fitted.

\subsection{Diagnostic for the Fitted Model}

We perform the diagnostic checking on the residuals of the fitted model by carrying out seasonal heteroscedasticity test and other complementary test such as Ljung-Box test for autocorrelation and Jarque-Bera test for normality $[15,16]$. This is done to validate that the fitted model is appropriate and it consists of the following.

\subsubsection{Seasonal Heteroscedasticity Test}

Thomas and William gave the likelihood ratio LR test that corresponds to a standard F-test for the seasonal heteroscedasticity null hypothesis as

$$
L R=-2\left(\ln \hat{L}_{0}-\ln \hat{L}_{1}\right)
$$

Where, $\hat{L}_{0}=$ maximized likelihood value from estimation of the restricted model, and $\hat{L}_{1}$ maximized likelihood value from estimation of the unrestricted model [22].

\subsubsection{Autocorrelation Test}

Ljung and Box recommend a test statistic which check whether the estimated residuals of the model behave approximately like a white noise [16]. The test statistic is as given below:

$$
Q(k)=n(n+2) \sum_{k=1}^{l} \frac{1}{n-k} \hat{r}(k)
$$




\subsubsection{Normality Test}

The Jarque and Bera is a goodness of the fit test of whether the residual estimated has the skewness and kurtosis matching a normal distribution and it is given as:

$$
\mathrm{JB}=\frac{n}{6}\left(S^{2}+\frac{1}{4}(k-3)^{2}\right)
$$

Where, $n=$ number of observations, $S=$ skewness, $k=$ kurtosis [15].

Forecasting

Once the parameters in PAR models have been estimated, one can use the resultant model for forecasting. Forecasting with PAR models starts the same way as with standard AR models [9]. Generally, it is more convenient to use vectors representation given in (5) to compute forecasts and forecast error variances.

The forecast for the one year ahead is given by

$$
\hat{Y}_{r+1}=E\left(Y_{r+1}\right)=E\left(\Phi_{0}^{-1} \Phi_{1} Y_{r}+\Phi_{0}^{-1} \in_{r+1}\right)=\Phi_{0}^{-1} \Phi_{1} Y_{r}
$$

The forecast error is $Y_{r}-\hat{Y}_{r}=\Phi_{0}^{-1} \in_{r+1}$ and the covariance matrix of the forecast error is $\sigma^{2}\left(\Phi_{0}^{-1}\left(\Phi_{0}^{-1}\right)^{\prime}\right)$

Likewise, the forecast for two years ahead is given by

$$
\hat{Y}_{r+2}=E\left(Y_{r+2}\right)=E\left(\left(\Phi_{0}^{-1} \Phi_{1}\right)^{2} Y_{r}+\Phi_{0}^{-1} \in_{r+2}+\left(\Phi_{0}^{-1} \Phi_{1}\right) \Phi_{0}^{-1} \in_{r+1}\right)=\left(\Phi_{0}^{-1} \Phi_{1}\right)^{2} Y_{r}
$$

The forecast error is $\Phi_{0}^{-1} \epsilon_{r+2}+\left(\Phi_{0}^{-1} \Phi_{1}\right) \Phi_{0}^{-1} \epsilon_{r+1}$ and the covariance matrix for the forecast error is given by $\sigma^{2}\left(\Phi_{0}^{-1}\left(\Phi_{0}^{-1}\right)^{\prime}+\left(\Phi_{0}^{-1} \Phi_{1} \Phi_{0}^{-1}\right)\left(\Phi_{0}^{-1} \Phi_{1} \Phi_{0}^{-1}\right)^{\prime}\right)$

\section{Results Discussion}

This section consists of presentation of results and interpretation.

Firstly, the researchers presented the time plot for the monthly tourism data, in order to visualize the pattern the data exhibited over the years under consideration (See figures 1 and 2).

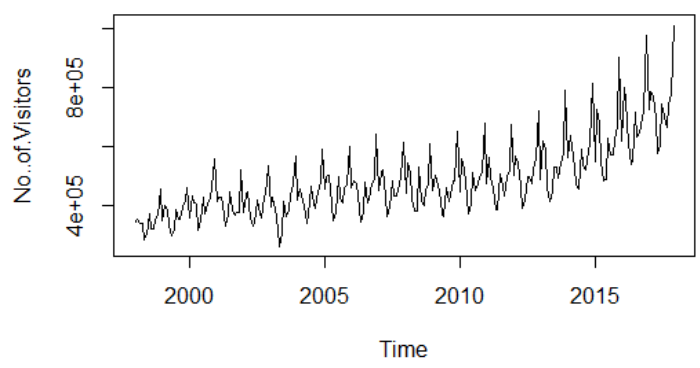

Figure 1. Time plot of Australian tourism data from 1998 to 2017.

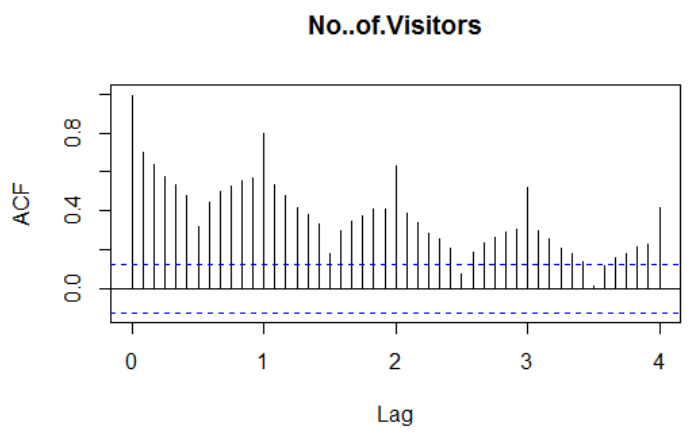

Figure 2. Correlogram of Australian tourism data from 1998 to 2017.

From the above figures, it was observed that the components in the tourism data (trend and seasonality) do not evolve independently. These suggest that periodic autoregressive time series models are suitable to model the tourism data. Periodic autoregressive PAR model was therefore fitted. The appropriate lag order of the PAR models was also determined using the BIC criteria in combination with Likelihood ratio test. The result is shown on Table 1 below.

Table 1. Periodic autoregressive order selection.

\begin{tabular}{lllll}
\hline \multirow{2}{*}{ Criterion } & \multicolumn{5}{l}{ Periodic autoregressive order } \\
\cline { 2 - 5 } & $\mathbf{1}$ & $\mathbf{2}$ & $\mathbf{3}$ & $\mathbf{4}$ \\
\hline $\mathrm{BIC}$ & -661.9366 & -664.2323 & -627.7320 & -587.4453 \\
$\mathrm{~F}\left(\varphi_{p+1, s}=0\right)$ & 5.6408 & 2.2593 & 1.8539 & 1.2022 \\
$\mathrm{p}$-value & 0.0000 & 0.0107 & 0.0431 & 0.2855 \\
\hline
\end{tabular}

From the result on Table 1, the p-value revealed that orders 1, 2 and 3 were significant based on the F-statistic value at 5percent significant level. Choosing the periodic autoregressive order among the significant lags, the BIC criterion having the lowest value of lag order was chosen. This implies that the order of the periodic autoregressive process for the tourism data is 2 .

Having identified the order for the PAR model suitable for the data, the periodicity in the autoregressive parameters of the model was verified to ensure there is periodic variation or periodicity before suggesting that $\operatorname{PAR}(2)$ is suitable. The periodic variation test result is summarized below for PAR with seasonal intercept and both seasonal intercept and seasonal trend.

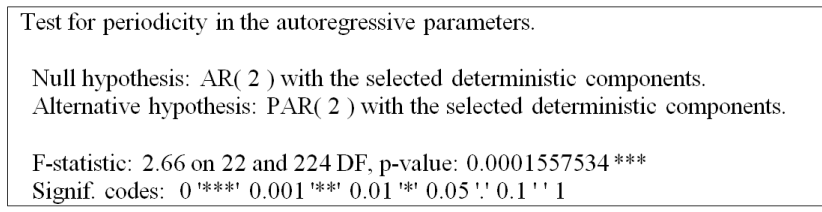

R console: See [19].

Figure 3. Test result for periodicity in the autoregressive parameters.

The result above showed that the periodicity was not rejected, meaning that a periodic model fits better to the tourism data rather than an autoregressive model, which is restrained to seasonally constant parameters.

The next step was to carry out property test to verify whether a unit root exists. If a unit root does not exist, then 
the periodic autoregressive process is stationary. On the other hand, if a unit root exists, then it suggests that the process will be either periodic autoregressive process for I(1) PARI or periodic integrated autoregressive PIAR to take account of the unit root.

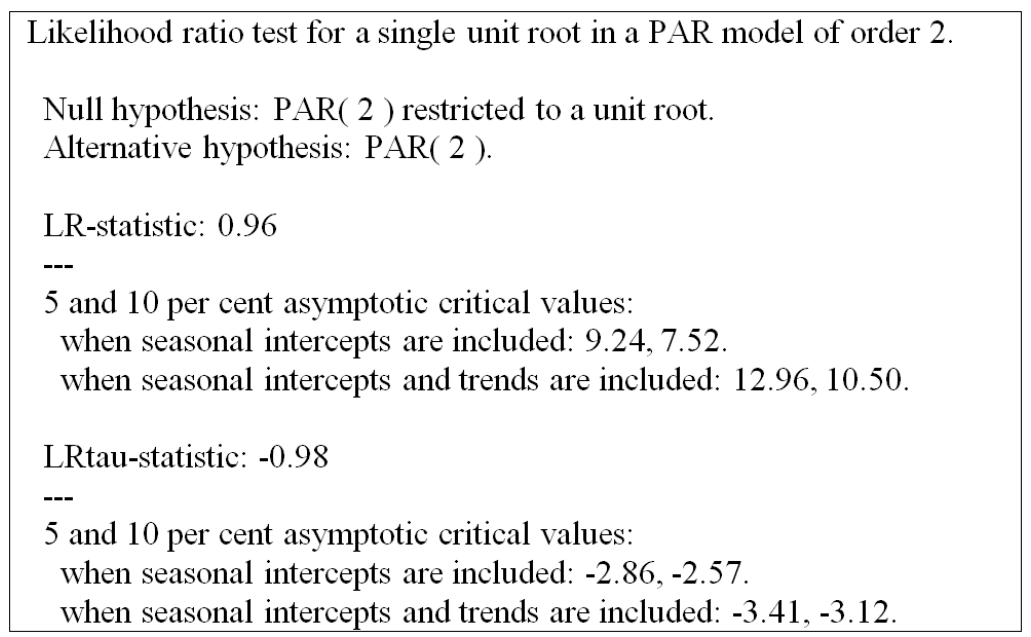

\section{$\mathrm{R}$ console.}

Figure 4. Result of LR test for a single unit root in a PAR model of order 2.

According to the LR test for a single unit root in a PAR model of order 2 above, the result showed that a single unit root cannot be rejected. This implies that a single unit root exists. To take account of it, it is worth verifying whether the parameters can be either 1 or -1 . This can be achieved by testing for a parameter restriction in a PAR model. If the hypothesis is accepted PARI process will be fitted, otherwise PIAR process will be appropriate.

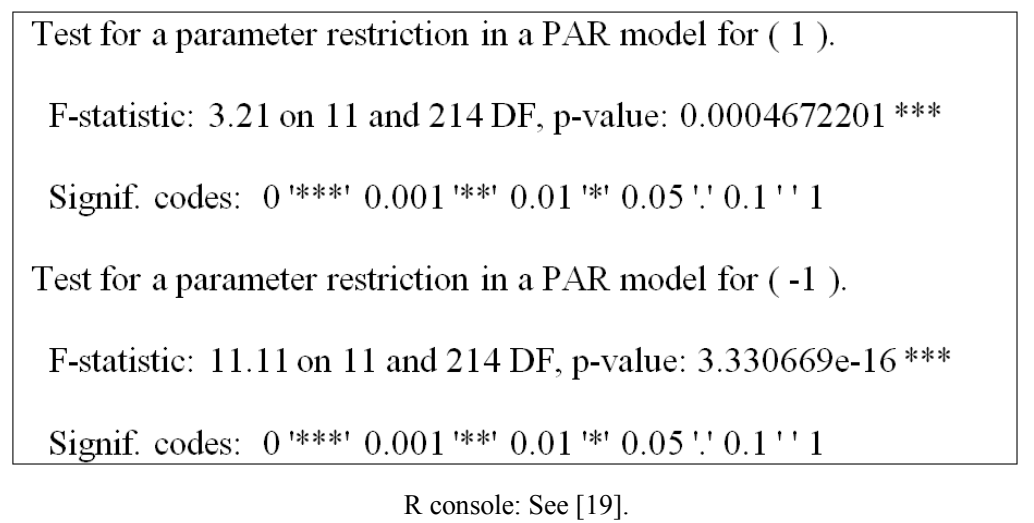

Figure 5. Result of test for a parameter restriction in a PAR(1).

According to the result, the hypothesis was rejected, hence, suggesting PIAR process to be appropriate for the data. Below is the graphical presentation of the periodical differenced data and summary of the estimated parameters of the PIAR(2) model.

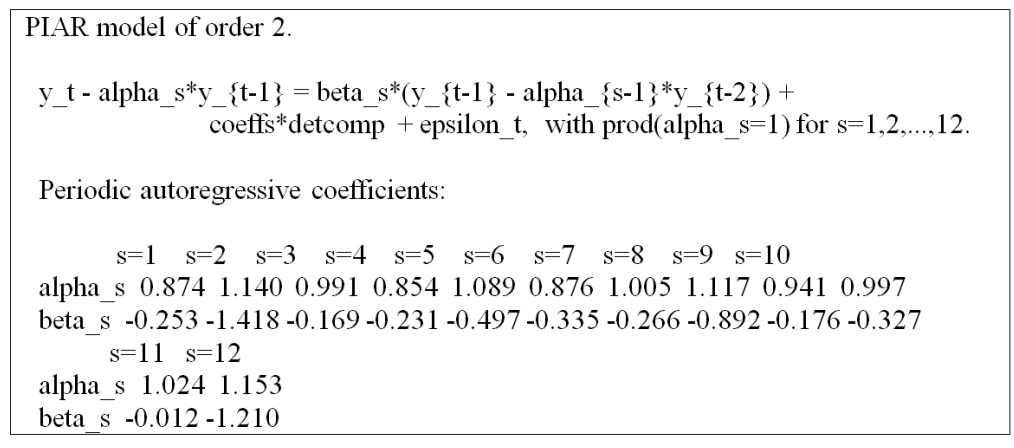

R console: See [19].

Figure 6. The coefficiients for PIAR model of order 2. 
Diagnostic checking to validate the model fitted for the data was performed considering seasonal heteroscedasticity, autocorrelation and normality tests.

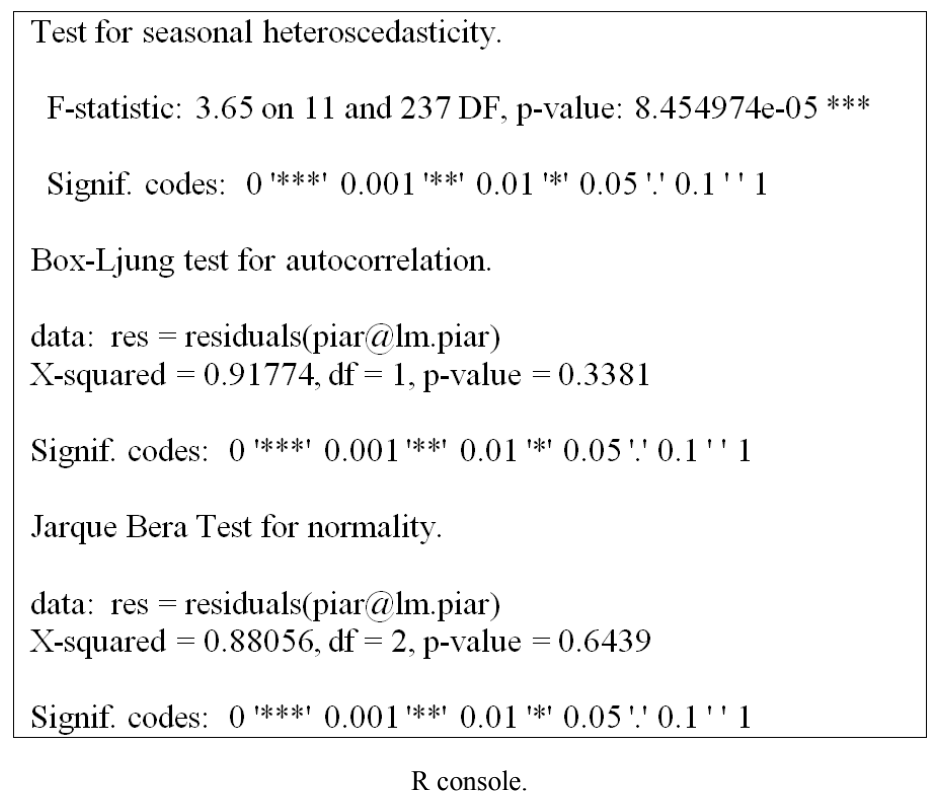

Figure 7. Result of test for seasonal heteroscedasticity.

The results of the diagnostic checking showed that seasonal heteroscedasticity was rejected. The Jarque-Bera normality test validates the normal distribution of the residuals; the Box-Ljung test for autocorrelation validates the presence of no autocorrelation of the residuals. All these verify that the model is adequate for the tourism data, hence, it can be used for forecasting.

\section{Conclusion}

Australian tourism was modeled using periodic autoregressive model. This was achieved by fitting a periodic autoregressive model, selecting the lag order of the model, testing for periodic variation or periodicity in the data, verifying if there is single unit root and periodic integration. From the results, it was revealed that the class of periodic autoregressive model called periodic integration autoregressive of order 2 PIAR(2) model is suitable for modelling Australian tourism. Based on this finding, it is concluded that periodic autoregressive time series model can be used to generate reliable forecast for Australian tourism data, and this could be attributed to the stochastic nature of the trend and seasonality associated with the time series data. However, we recommend that for further studies.

\section{Conflict of Interest}

There is no conflict of interest among the authors.

\section{References}

[1] Australian National Accounts: Tourism Satellite Account.; Reference period: 2014-15 financial year.
[2] Australian National Accounts: Tourism Satellite Account; Reference period: 2019-20 financial year.

[3] Boswijk, H. P. \& Frances, P. H. (1996). Unit roots in Periodic Autoregressions. Journal of Time Series Analysis, 17, 221-245.

[4] Boswijk, H. P., Frances, P. H., \& Haldrup, N. (1997). Multiple Unit roots in Periodic Autoregression. Journal of Econometrics, 80, 167-193.

[5] Box, G. E. P. \& Jenkins, G. M. (1970). Time Series Analysis: forecasting and Control. Holden-Day, San Francisco.

[6] Eugen, U. \& Pereau, J. C. (2016). Application of periodic autoregressive process to the modeling of the Garonne river flows. Published in Stochastic Environmental Research and Risk Assessment, Springer Verlag (Germany), inPress, 30 (7), pp. 1785-1795. Doi: 10.1007/s00477-015-1193-3.

[7] Eugen, U. \& Pereau, J. C. (2017). Estimation and identification of periodic autoregressive models with one exogenous variable. Journal of Statistical Society. 46, 629640 .

[8] Frances, P. H. \& Paap, R. (1996). Periodic Integration: Further results on Model Selection and Forecasting. Statistical paper, $37,33-52$.

[9] Franses, P. (1996), Periodicity and Stochastic Trends in Economic Time Series, Advanced Texts in Econometrics. Oxford University Press.

[10] Franses, P. H. \& Paap, R. (2004), Periodic Time Series Models, Advanced Texts in Econometrics. Oxford University Press.

[11] Harvey, A. C. (1989). Forecasting Structural time series and Kalman Filter. Cambridge University Press, Cambridge.

[12] Herwartz, H. (1997). Performance of Periodic Error Correction models in Forecasting Consumption Data. International Journal of Forecasting. 13, 421-431. 
[13] Hipel, K. W. and McLeod, A. I. (1994). Time Series Modelling of Water Resources and Environmental Systems. Vol. 45, pp iii-xxxvii, 1-1013 (1994), Elsevier Science Publishers.

[14] Hylleberg, S., Engle, R. F., Granger, C. W. J. \& Yoo, B. (1990). Seasonal Integration and Cointegration. Journal of Econometrics, 44, 215-238.

[15] Jarque CM, Bera AK (1987) A test for normality of observations and regression residuals. Int Stat Rev 55 (2): 163-172.

[16] Ljung, G. M. and Box, G. E. P. (1978). On a Measure of Lack of Fit in Time Series Models. Biometrika 65 (2); 297-303 DOI: 10.1093/biomet/65.2.297.

[17] Lopez-de Lacalle, J. (2005), 'Periodic autoregressive time series models in R: The partsm package', BILCODEC 2005 working paper, Universidad del Pas Vasco UPV/EHU Departamento de Economa Aplicada III (Econometray Estadstica). URL:

http://econpapers.repec.org/software/ehubilcod/200501. htm.

[18] Novales, A. \& Flores de Fruto, R. (1997). Forecasting with Periodic Models: A comparison with time Invariant Coefficient Models. International Journal of Forecasting. 13, 393-405.
[19] Osborn, D. R. (1988). Seasonality and Habit Persistence in a Life Cycle Model of consumption. Journal of Applied Econometrics, 3, 255-266.

[20] Osborn, D. R. \& Smith, J. P. (1989). The Performance of Periodic Autoregressive Models in Forecasting Seasonal U. K. Consumption. Journal of Business \& Economic Statistics, 7, 117-127.

[21] R Development Core Team (2011), R: A language and environment for statistical computing, $\mathrm{R}$ Foundation for Statistical Computing, Vienna, Austria. ISBN 3-900051-07-0. URL: http://www.R-project.org.

[22] Thomas, T. and William, B. (2006). Models for seasonal heteroskedastic time series estimation, testing and simulation evidence, Manuscript.

[23] Tourism definition according to https://www.go2hr.ca

[24] Ursu, E. and Duchesna, P. (2009). On modeling and diagnostic checking of vector periodic autoregressive time series models. Journal of Time Series Analysis 30 (1): 70-96.

[25] Wells, J. M. (1997). Modelling Seasonal Pattern and LongRun Trends in U.S. Time Series. International Journal of Forecasting, 13, 405-418. 